\title{
Characterisation of phenotypes based on severity of emphysema in chronic obstructive pulmonary disease
}

\author{
Hironi Makita, Yasuyuki Nasuhara, Katsura Nagai, Yoko Ito, Masaru Hasegawa, Tomoko \\ Betsuyaku, Yuya Onodera, Nobuyuki Hizawa, Masaharu Nishimura, the Hokkaido COPD Cohort \\ Study Group*
}

See end of article for authors' affiliations

Correspondence to: Dr Masaharu Nishimura First Department of Medicine, Hokkaido University School of Medicine, N-15 W-7, Kita$\mathrm{Ku}$, Sapporo 060-8638, Japan; ma-nishi@med.

hokudai.ac.jp

Received 4 October 2006 Accepted 17 April 2007 Published Online First 15 June 2007

\begin{abstract}
Background: Airflow limitation in chronic obstructive pulmonary disease (COPD) is caused by a mixture of small airway disease and emphysema, the relative contributions of which may vary among patients. Phenotypes of COPD classified purely based on severity of emphysema are not well defined and may be different from the classic phenotypes of "pink puffers" and "blue bloaters".

Methods: To characterise clinical phenotypes based on severity of emphysema, 274 subjects with COPD were recruited, excluding those with physician-diagnosed bronchial asthma. For all subjects a detailed interview of disease history and symptoms, quality of life (QOL) measurement, blood sampling, pulmonary function tests before and after inhalation of salbutamol $(0.4 \mathrm{mg})$ and high-resolution CT scanning were performed.

Results: Severity of emphysema visually evaluated varied widely even among subjects with the same stage of disease. No significant differences were noted among three groups of subjects classified by severity of emphysema in age, smoking history, chronic bronchitis symptoms, blood eosinophil count, serum lgE level or bronchodilator response. However, subjects with severe emphysema had significantly lower body mass index (BMI) and poorer QOL scores, evaluated using St George's Respiratory Questionnaire (SGRQ), than those with no/mild emphysema (mean (SD) BMI $21.2(0.5)$ vs $23.5(0.3) \mathrm{kg} / \mathrm{m}^{2}$, respectively; SGRQ total score 40 (3) vs 28 (2), respectively; $p<0.001$ for both). These characteristics held true even if subjects with the same degree of airflow limitation were chosen.

Conclusions: The severity of emphysema varies widely even in patients with the same stage of COPD, and chronic bronchitis symptoms are equally distributed irrespective of emphysema severity. Patients with the phenotype in which emphysema predominates have lower BMI and poorer health-related QOL.
\end{abstract}

$\mathrm{C}$ hronic obstructive pulmonary disease (COPD) is the fourth leading cause of death worldwide, ${ }^{1}$ and morbidity and mortality has been increasing in $\mathrm{Japan}^{2}$ as in many other Western countries. ${ }^{3}$ COPD is described as a disease state characterised by airflow limitation that is not fully reversible according to the Global Initiative for Chronic Obstructive Lung Disease (GOLD) guidelines and the American Thoracic Society/ European Respiratory Society consensus guidelines. ${ }^{145}$ This airflow limitation is progressive and caused by a mixture of abnormal inflammatory responses in small airways and parenchymal destruction of the lungs, the relative contributions of which vary from person to person. ${ }^{6}$

Historically, typical phenotypes of COPD used to be known as "pink puffers" and "blue bloaters", ${ }^{78}$ or A, B and X types. ${ }^{9}$ This is because COPD had been recognised as a disease that is a mixture of chronic bronchitis and emphysema, with predominantly bronchitis and predominantly emphysema as the two extreme phenotypes. ${ }^{10}$ However, a number of studies over the last three decades have revealed small airways as the most important site causing airflow limitation in COPD, ${ }^{11-14}$ and parenchymal destruction (emphysema) is certainly a contributing factor to a variable extent through the loss of elastic recoil pressure. ${ }^{14}{ }^{5}$ Hogg et al recently re-demonstrated how important inflammatory changes in small airways are as a determinant of progression and severity in COPD. ${ }^{15}$ The narrowing of small airways caused by inflammation and scarring and the blocking of small airway lumens with mucous secretions are thought to represent the primary pathology of airflow limitation. ${ }^{6}$

* See end of article for all contributors to the Hokkaido COPD Cohort Study
In this study we therefore examined the clinical phenotype based purely on the severity of emphysema quantitatively evaluated using high-resolution CT (HRCT) scanning. If the severity of emphysema varies despite the same degree of airflow limitation, subjects with COPD can be compared where the relative contributions of small airway disease and emphysema vary. For instance, subjects displaying little evidence of emphysema despite severe airflow limitation could be considered as showing a phenotype with predominantly small airway disease but not necessarily that of chronic bronchitis. This is the first report from the Hokkaido COPD cohort study, which was primarily designed to evaluate the natural history and prognosis of COPD as classified by severity of emphysema.

\section{METHODS}

\section{Subjects}

A total of 307 subjects with physician-diagnosed COPD were recruited at Hokkaido University Hospital and nine affiliated hospitals from May 2003 to the end of April 2005. All study protocols were approved by the ethics committees of all hospitals and all subjects provided written informed consent. All were either current or former smokers with a smoking history of at least 10 pack-years. Subjects diagnosed by respiratory physicians as having bronchial asthma or

Abbreviations: $B D R$, bronchodilator response; $B M I$, body mass index; $\mathrm{COPD}$, chronic obstructive pulmonary disease; $\mathrm{FEV}_{1}$, forced expiratory volume in $1 \mathrm{~s} ; \mathrm{FVC}$, forced vital capacity; HRCT, high-resolution computed tomography; LAA, low attenuation area; $Q O L$, quality of life; SGRQ, St George's Respiratory Questionnaire; TLCO, carbon monoxide transfer factor; $\mathrm{VA}$, alveolar volume 
bronchiectasis at entry to the study were excluded. Subjects were also excluded if they had active tuberculosis, any history of lung cancer, any history of lung resection, and any history of cystic fibrosis, allergic alveolitis or pulmonary fibrosis. Those who would compromise 5-year follow-up or accurate assessment of pulmonary function or who were receiving long-term supplemental oxygen therapy for $>12 \mathrm{~h} /$ day were also excluded. To avoid interference with bronchodilator reversibility testing, those who had been taking non-selective $\beta$ blockers for treatment of hypertension were excluded. On the first visit, diagnoses were reconfirmed by pulmonologists and established based on the spirometric criteria of the GOLD guidelines. ${ }^{1}$ While 33 subjects were excluded from this study because the post-bronchodilator ratio of forced expiratory volume in $1 \mathrm{~s}\left(\mathrm{FEV}_{1}\right)$ to forced vital capacity (FVC) was $\geqslant 0.7$, these subjects were encouraged to join the subsequent followup study.

\section{Chronic bronchitis symptoms}

Well trained clinical research coordinators elicited disease history, smoking history and other information about all treatments. Chronic cough and sputum expectoration were considered to be present when they occurred on most days for $\geqslant 3$ months/year and for $\geqslant 2$ consecutive years. ${ }^{16}{ }^{17}$ To avoid a bias by patient reports about the presence of chronic sputum symptoms, the amount of sputum should be $>10 \mathrm{ml} /$ day for the definition described above and this was confirmed by clinical research coordinators for all subjects.

\section{Pulmonary function tests}

Rolling seal spirometers (Chestac; Chest MI Inc, Tokyo) or Fudac (Fukuda Denshi Co Ltd, Tokyo) were used for the spirometric measurements and carbon monoxide transfer factor (TLCO) at all hospitals. Further details of the procedures are provided in the online supplement available at http://thorax. bmj.com/supplemental. Predicted values of spirometric measurements were derived from the guidelines for pulmonary function tests issued by the Japanese Respiratory Society. ${ }^{18}$ TLCO was measured by the single breath method. Results were corrected by alveolar volume (VA) and haemoglobin concentration. TLCO/VA values were compared with the predicted normal values. ${ }^{18}$

The reversibility of airflow limitation was evaluated by measuring spirometry before and $30 \mathrm{~min}$ after inhalation of salbutamol $(0.4 \mathrm{mg})$. The bronchodilator response (BDR) was expressed in three ways: (1) as an absolute change in $\mathrm{FEV}_{1},(2)$ as a percentage change from baseline $\mathrm{FEV}_{1}$, and (3) as a percentage change from predicted $\mathrm{FEV}_{1}$. Reversibility of airflow limitation was considered to be significant if an increase in $\mathrm{FEV}_{1}$ was both $>200 \mathrm{ml}$ and $12 \%$ above pre-bronchodilator $\mathrm{FEV}_{1}$ according to GOLD guidelines. ${ }^{1}$

\section{HRCT scanning}

Chest HRCT scans were performed in the supine position with the breath held at full inspiration. CT scanners used in nine hospitals are described in the online supplement available at http://thorax.bmj.com/supplemental. Technical parameters were as follows: $1 \mathrm{~mm}$ collimation, $120-140 \mathrm{kV}, 75-350 \mathrm{~mA}$, 0.75-1 s scanning time and $1-2 \mathrm{~mm}$ thickness. HRCT images were selected at three levels including the aortic arch, carina and $1-2 \mathrm{~cm}$ above the highest hemidiaphragm. Image interpretations were performed under -600 to -900 Hounsfield units (HU) window levels and 800-1500 HU window widths based on the best condition for detecting emphysema at each hospital.

The severity of emphysema was visually assessed by three independent pulmonologists according to the modified
Goddard scoring system. ${ }^{19}$ Six images were analysed in three slices in the lungs and an average score of all images was considered as a representative value of the severity of emphysema in each person. Each image was classified as normal (score 0$), \leqslant 5 \%$ affected (score 0.5 ), $\leqslant 25 \%$ affected (score 1 ), $\leqslant 50 \%$ affected (score 2 ), $\leqslant 75 \%$ affected (score 3 ) and $>75 \%$ affected (score 4 ), giving a minimum score of 0 and maximum of 4 . When the three independent pulmonologists disagreed in their evaluation, only the score assessed by the majority was taken.

Three-dimensional CT analyses were performed only in Hokkaido University Hospital $(n=137)$ to confirm the accuracy and reliability of visual assessment. The method of computerised assessment of emphysema for the whole lung is described in detail in the online supplement available at http://thorax.bmj.com/supplemental.

\section{St George's Respiratory Questionnaire (SGRQ)}

The SGRQ was used to assess health-related QOL. This is a supervised self-administered measure designed specifically for use in respiratory disease and contains three domains: symptoms (relating to cough, sputum, wheeze and shortness of breath); activity (relating to physical activity limited by breathlessness); and impact (relating to control, panic, medication and expectations). ${ }^{20} \mathrm{~A}$ total score was calculated from all three domains.

\section{Blood samples}

Blood was taken from all subjects for the measurement of $\alpha_{1^{-}}$ antitrypsin, leucocyte count, eosinophil count and immunoglobulin (Ig)E levels.

\section{Statistical analysis}

Data are shown as mean (SE) values unless otherwise specified. Skewed data were either transformed to logarithmic data or expressed as median values with interquartile range (IQR). Univariate analysis used $\chi^{2}$ tests for categorical variables and one-way analysis of variance for quantitative variables with Scheffe's test as a post-hoc test for multiple comparisons. Relationships between two variables of quantitative data were examined using Spearman tests. For BMI and SGRQ scores, the

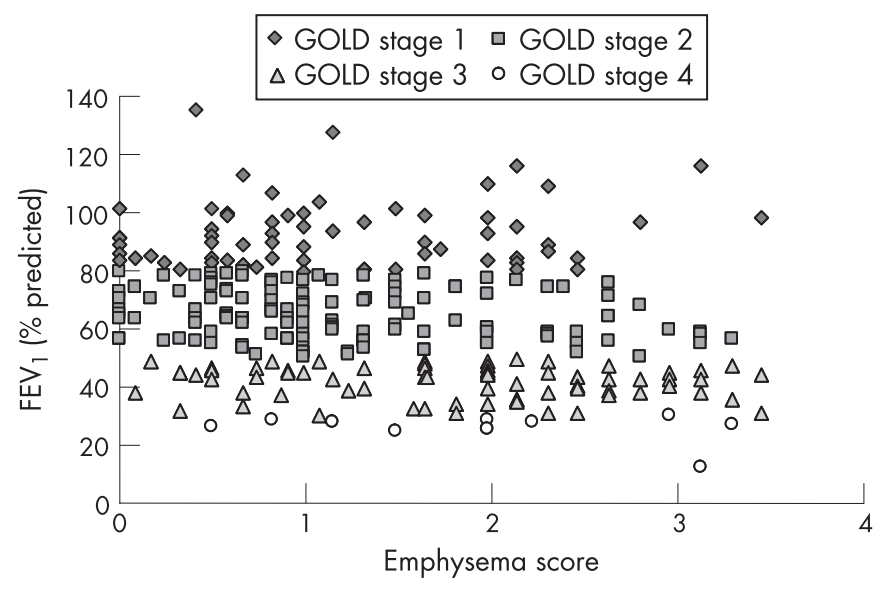

Figure 1 Relationship between emphysema score and forced expiratory volume in $1 \mathrm{~s}\left(\mathrm{FEV}_{1}\right) \%$ predicted in 274 subjects with chronic obstructive pulmonary disease (COPD). They were classified into four stages according to the Global Initiative for Chronic Obstructive Lung Disease (GOLD) guidelines (stage 1, $n=64$; stage $2, n=127$; stage $3, n=71$; and stage 4, $n=12$ ). Severity of emphysema was highly variable despite the same stage of COPD, although the relationship between emphysema score and postbronchodilator $\mathrm{FEV}_{1} \%$ predicted was significant overall ( $r=-0.302$, $\mathrm{p}<0.001$ ). 
Table 1 Clinical characteristics of phenotypes based on severity of emphysema*

\begin{tabular}{|c|c|c|c|c|}
\hline Characteristic & $\begin{array}{l}\text { No/mild } \\
(\mathrm{n}=105)\end{array}$ & $\begin{array}{l}\text { Moderate } \\
(n=124)\end{array}$ & $\begin{array}{l}\text { Severe } \\
(n=45)\end{array}$ & $\begin{array}{l}\text { Total } \\
(n=274)\end{array}$ \\
\hline Age (years) & $70(64-76)$ & $72(64-75)$ & 71 (68-77) & $71(64-76)$ \\
\hline Sex (male/female) & $96 / 9$ & $119 / 5$ & $43 / 2$ & $258 / 16$ \\
\hline BMI $\left(\mathrm{kg} / \mathrm{m}^{2}\right)$ & $23.5(0.3)$ & $21.7(0.3) \dagger$ & $21.2(0.5) \dagger$ & $22.3(0.2)$ \\
\hline Smoking (pack-years) & $58(3)$ & $65(3)$ & $66(4)$ & $63(2)$ \\
\hline Current smoker (\%) & 39/105 (37) & $26 / 124(21)$ & $10 / 45(22)$ & $75 / 274(27)$ \\
\hline \multicolumn{5}{|l|}{ Clinical symptoms (\%) } \\
\hline Chronic cough & 11/105 (11) & 18/124 (15) & $5 / 45(11)$ & $34 / 274(12)$ \\
\hline Chronic sputum & 19/105 (18) & $23 / 124$ (19) & $7 / 45(16)$ & $49 / 274(18)$ \\
\hline Chronic cough and sputum & $8 / 105(8)$ & $16 / 124(13)$ & $5 / 45(11)$ & $29 / 274(11)$ \\
\hline \multirow{2}{*}{\multicolumn{5}{|c|}{$\begin{array}{l}\text { Pulmonary function tests } \\
\text { Pre-bronchodilator }\end{array}$}} \\
\hline & & & & \\
\hline FVC (\% predicted) & $91.4(2.0)$ & $94.7(2.0)$ & $88.3(3.1)$ & $92.4(1.3)$ \\
\hline $\mathrm{FEV}_{1}$ (\% predicted) & $63.0(2.0)$ & $56.9(2.1) \dagger$ & $46.5(3.0) \dagger \ddagger$ & $57.5(1.4)$ \\
\hline \multicolumn{5}{|l|}{ Post-bronchodilator } \\
\hline FVC (\% predicted) & $98.9(1.8)$ & $102.6(1.8)$ & $98.4(2.9)$ & $100.5(1.2)$ \\
\hline $\mathrm{FEV}_{1}$ (\% predicted) & 69.5 (1.9) & $62.7(2.0)$ & $52.0(3.0) \dagger \ddagger$ & $63.5(1.3)$ \\
\hline $\mathrm{FEV}_{1} / \mathrm{FVC}$ & $0.56(0.01)$ & $0.48(0.01) \dagger$ & $0.42(0.02) \dagger \ddagger$ & $0.50(0.01)$ \\
\hline \multicolumn{5}{|l|}{ Transfer factor } \\
\hline TLCO/VA (\% predicted) & $78.1(2.2)$ & $58.4(1.8) \dagger$ & $41.6(2.5) \dagger \ddagger$ & $63.3(1.5)$ \\
\hline \multicolumn{5}{|l|}{ Blood analysis } \\
\hline Eosinophils (log cells/ $\mu$ l) & $2.26(0.03)$ & $2.16(0.03)$ & $2.19(0.05)$ & $2.20(0.02)$ \\
\hline Serum total $\lg E(\log \mathrm{IU} / \mathrm{ml})$ & $1.87(0.07)$ & $1.72(0.06)$ & $1.74(0.09)$ & $1.78(0.04)$ \\
\hline$\alpha_{1}$-antitrypsin $(\mathrm{mg} / \mathrm{dll})$ & $128(2)$ & $129(2)$ & $135(3)$ & $129(1)$ \\
\hline \multicolumn{5}{|l|}{$\begin{array}{l}\text { Medications, no. of patients/ } \\
\text { total (\%) }\end{array}$} \\
\hline Anticholinergic agents & $41 / 105$ (39) & $64 / 124(52)$ & 32/45 (71)†‡ & $137 / 274(50)$ \\
\hline$\beta_{2}$ agonists & $29 / 105(28)$ & $43 / 124$ (35) & $18 / 45(40)$ & $90 / 274(33)$ \\
\hline Theophyllines & $36 / 105$ (34) & $59 / 124(48) \dagger$ & $27 / 45(60) \dagger$ & $122 / 274(45)$ \\
\hline ICS & 12/105 (11) & $10 / 124(8)$ & $9 / 45(20)$ & $31 / 274(11)$ \\
\hline \multicolumn{5}{|c|}{$\begin{array}{l}\text { BMI, body mass index; FVC, forced vital capacity; } \mathrm{FEV}_{1} \text {, forced expiratory volume in } 1 \mathrm{~s} \text {; TLCO, carbon monoxide } \\
\text { transfer factor; } \mathrm{VA}_{\mathrm{A}} \text {, alveolar volume; ICS, inhaled corticosteroids; no/mild, no or mild emphysema (emphysema score } \\
<1 \text { ); moderate, moderate emphysema (emphysema score } \geqslant 1-<2.5 \text { ); severe, severe emphysema (emphysema score } \\
\leqslant 2.5 \text { ). } \\
\text { *Data shown as mean (SE) values except for skewed data which are expressed as median (interquartile range). } \\
\text { Univariate analysis used } \chi^{2} \text { tests for categorical variables and one-way analysis of variance for quantitative variables } \\
\text { with Scheffe's test as a post-hoc test for multiple comparisons. } \\
\dagger p<0.05 \text { vs no/mild emphysema; } \neq p<0.05 \text { vs moderate emphysema. }\end{array}$} \\
\hline
\end{tabular}

Jonckheere-Terpstra test was used to examine trends for three groups of subjects. All statistical tests were two-sided and values of $\mathrm{p}<0.05$ were considered statistically significant. Data were analysed using SPSS for Windows Version 12 (SPSS Japan, Tokyo).

\section{RESULTS}

There was a high degree of correlation between the subjective visual score of severity of emphysema for three CT images and an objective computerised quantification for the whole lung ( $\mathrm{n}=137, r=0.835, \mathrm{p}<0.0001)$ which was performed only for the subjects in Hokkaido University Hospital. This therefore justifies the use of visual assessment of emphysema for this multi-site study.

Figure 1 shows the relationship between the emphysema score and post-bronchodilator value of $\mathrm{FEV}_{1} \%$ predicted in all subjects with COPD. A weak but significant overall correlation was seen between the two parameters $(n=274, r=-0.302$, $\mathrm{p}<0.001)$. However, a better correlation was seen between the emphysema score and TLCo/VA $(\mathrm{n}=273, r=-0.577, \mathrm{p}<0.001)$. An extremely wide variation in the severity of emphysema was seen with all stages of COPD. In other words, the severity of emphysema varied widely from none/mild to very severe even in subjects with the same level of airflow limitation. A similar finding was noted even if those subjects showing significant reversibility of airflow limitation $(n=86)$ as defined by the GOLD guidelines were excluded (data not shown).

To emphasise the characterisation of phenotypes in COPD, all subjects were then classified into three groups based on severity of emphysema: (1) subjects with no/mild emphysema (emphysema score $<1$, percentage of low attenuation area (LAA) in the assessed lung $<12.5 \%$ on average); (2) subjects with moderate emphysema (emphysema score $\geqslant 1-<2.5$, percentage of LAA in the assessed lung $<50 \%$ on average); and (3) subjects with severe emphysema (emphysema score $\geqslant 2.5$ ). Table 1 shows the characteristics of the three groups. Although indices of airflow limitation and TLCo deteriorated as emphysema became more severe, no significant differences in age, sex, smoking history, blood eosinophil count or serum IgE levels were found. There was no $\alpha_{1}$-antitrypsin deficiency in any subjects, and no significant difference was noted in the mean level of serum $\alpha_{1}$-antitrypsin among the three groups. In terms of medication, anticholinergic agents or theophyllines were prescribed more often as emphysema became more severe, however $\beta_{2}$ agonists and inhaled corticosteroids were given to a similar extent in all three groups of subjects. The prevalence of chronic cough and/or sputum was remarkably similar among the three groups, indicating that the prevalence of chronic bronchitis is the same regardless of the severity of emphysema.

In terms of BDR to salbutamol, there was wide variation between subjects but a clear relationship was apparent between baseline $\mathrm{FEV}_{1}$ and post-bronchodilator increase in $\mathrm{FEV}_{1}$ when expressed as a percentage change from baseline $\mathrm{FEV}_{1}$ (fig 2A). BDR was then compared among the three groups classified according to severity of emphysema. No significant differences were seen in absolute change in $\mathrm{FEV}_{1}$ (no/mild emphysema, 173 (13) ml $(\mathrm{n}=105)$; moderate emphysema, 163 (12) ml $(\mathrm{n}=123)$; severe emphysema,150 (20) ml $(\mathrm{n}=45))$, percentage change from baseline $\mathrm{FEV}_{1}(12.6$ (1.2)\%, 14.1 (1.3)\% and $14.1(2.0) \%$, respectively; fig $2 \mathrm{~B})$ or percentage change from predicted $\mathrm{FEV}_{1}(6.5(0.5) \%, 6.1 \quad(0.4) \%$ and $5.6 \quad(0.7) \%$, 

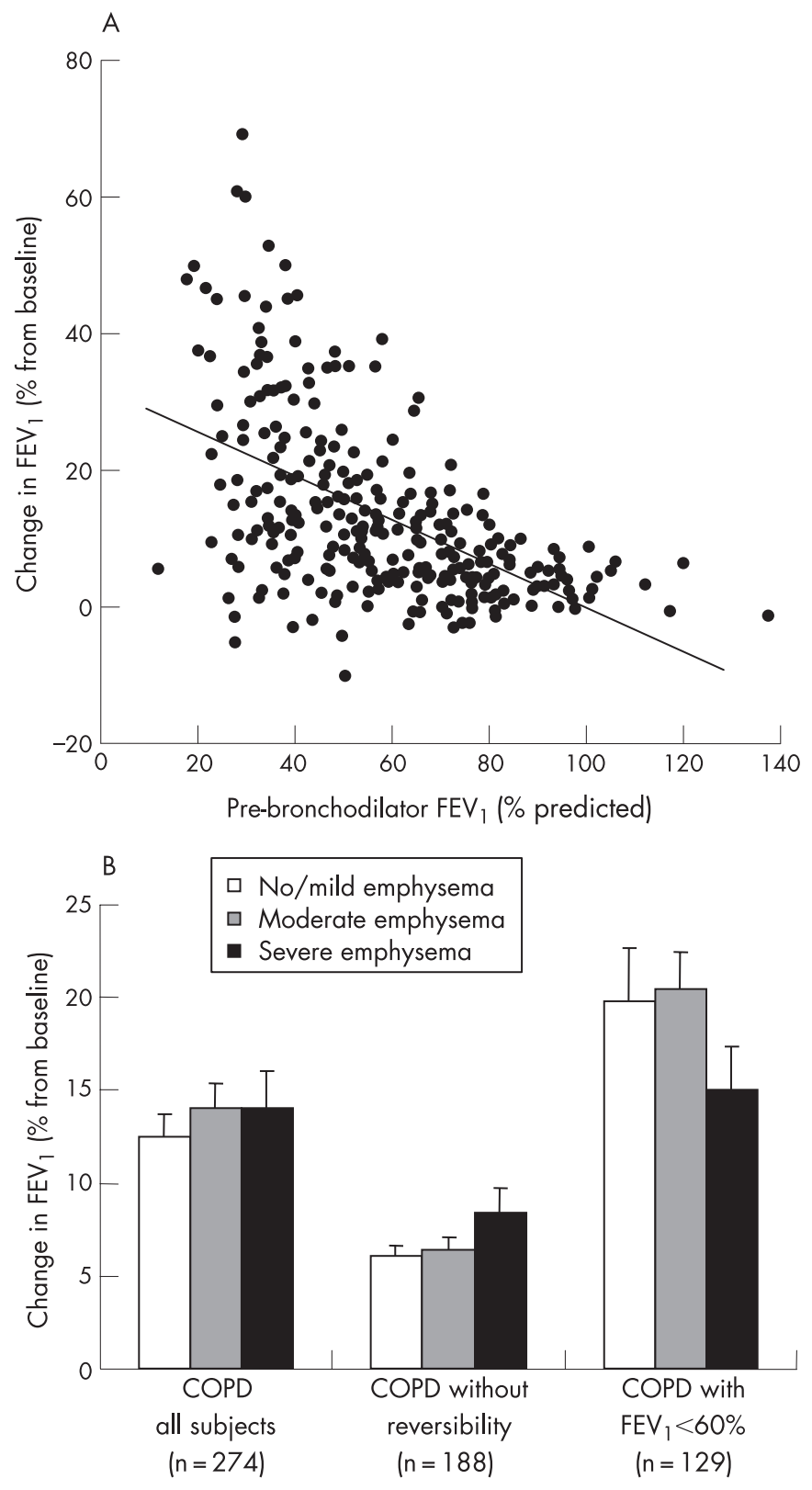

Figure 2 (A) Relationship between pre-bronchodilator forced expiratory volume in $1 \mathrm{~s}\left(\mathrm{FEV}_{1}\right)$ and bronchodilator response (BDR) to $\beta_{2}$ agonist in all subjects with chronic obstructive pulmonary disease. (B) Comparison of BDR among three groups classified by severity of emphysema. Values are mean (SE).

respectively). Significant differences between the groups were still absent even if we chose only subjects without reversibility of airflow limitation or those with $\mathrm{FEV}_{1}<60 \%$ predicted, indicating that airflow limitation was perfectly comparable between the three groups (post-bronchodilator $\mathrm{FEV}_{1}$ in no/mild emphysema, 46.9 (1.6)\% predicted $(\mathrm{n}=32)$; moderate emphysema, 44.8 (1.3)\% predicted $(\mathrm{n}=63)$; severe emphysema, 43.5 (1.7)\% predicted $(\mathrm{n}=35))$.

Figure 3 shows data for BMI and health-related QOL. BMI was significantly lower as emphysema became more severe in all subjects (table 1). Of particular note is the fact that this held true even if subjects were compared separately based on the COPD stage (fig $3 \mathrm{~A}$ ) or only subjects with $\mathrm{FEV}_{1}<60 \%$ predicted, indicating that airflow limitation was perfectly comparable between the three groups classified by the severity of emphysema (no/mild emphysema, 23.7 (0.6) kg/m², $\square \mathrm{No} /$ mild emphysema

$\square$ Moderate emphysema

- Severe emphysema
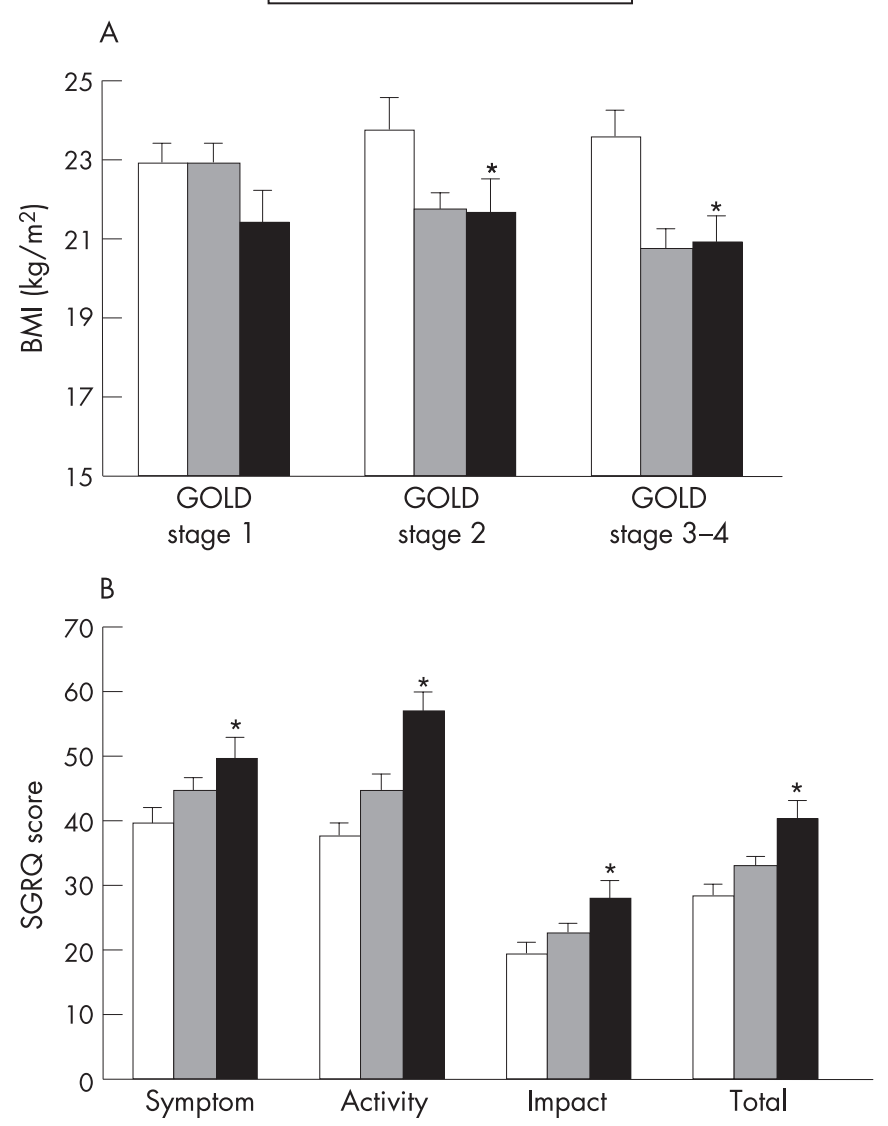

Figure 3 (A) Body mass index (BMI) and (B) St George's Respiratory Questionnaire (SGRQ) in the three groups classified by severity of emphysema. Values are mean (SE). Severity of emphysema is associated with lower BMI regardless of GOLD stage. See text for details of SGRQ data. The Jonckheere-Terpstra test was used to examine trends for the three groups of subjects ( $\left.{ }^{*} p<0.05\right)$.

moderate emphysema, $21.1(0.4) \mathrm{kg} / \mathrm{m}^{2}$; severe emphysema, $21.1(0.6) \mathrm{kg} / \mathrm{m}^{2}$ ). All dimensions of SGRQ scores became significantly higher as emphysema became more severe (fig 3B). Statistical differences remained present in the activity and total scores of the SGRQ even for subjects with $\mathrm{FEV}_{1}<60 \%$ predicted (activity score, 51 (4)\%, $52(3) \%$ and $64(3) \%$, respectively; total score, $37(3) \%, 40(2) \%, 45(2) \%$, respectively). There was therefore a significant relationship between severity of emphysema and BMI overall $(r=-0.293, \mathrm{p}<0.001)$ and also with the SGRQ total score $(r=0.231, \mathrm{p}<0.001)$.

\section{DISCUSSION}

This study has shown that the severity of emphysema varies widely despite the same disease stage in COPD. For instance, even in patients with moderate or severe COPD, some show very little evidence of emphysema while others have marked emphysema. Furthermore, some patients retain relatively normal pulmonary function despite the presence of severe emphysema. These observations support the findings of several past studies which argued against emphysema as the major cause of airflow limitation in COPD. ${ }^{121-23}$

All subjects were then classified into three groups based on the severity of emphysema. Although the small airways were not directly evaluated in this study, the extremely wide variation in severity of emphysema observed among subjects 
with the same degree of airflow limitation appeared to indicate that the three groups might well represent differences in relative contributions of small airway disease and emphysema to airflow limitation. The most important findings in this study were that patients with severe emphysema had significantly lower BMI and worse QOL than patients with no/mild emphysema (predominantly small airway disease), despite similarities in age, smoking history, blood eosinophil count and IgE levels. Activity score, which includes dyspnoea on exertion, was particularly significant. These differences remained significant even if subjects with the same degree of airflow limitation were compared. Celli et al ${ }^{24}$ recently proposed the BODE index, a simple multidimensional grading system, for predicting the risk of death in subjects with COPD. They demonstrated the importance of BMI, dyspnoea and exercise capacity index for assessment of subjects with COPD in addition to airflow limitation index. Several other reports support the notion that BMI and QOL, including dyspnoea, represent independent factors for the prognosis of COPD..$^{25-28}$

Phenotyping of COPD described in the present study may thus have some clinical relevance in the management of patients with COPD. Another important consideration is that more attention should be paid to these phenotypes when studying the epidemiology, genetic background and pathogenesis of COPD..$^{29}$ In fact, an interesting study from Japan recently showed that body weight loss in COPD is associated with a novel polymorphism in secretory $\mathrm{A}_{2}$-IID, an enzyme responsible for mobilisation of fatty acids including arachidonic acid from phospholipids, thereby potentially influencing systemic inflammation in COPD. ${ }^{31}$ Possible reasons why severity of emphysema rather than airflow limitation itself is associated with lower BMI may be exaggerated systemic inflammatory response or increased work load of breathing in the emphysematous type of COPD; however, these speculations are beyond the scope of this study and need further investigation.

Historically, patients with COPD used to be classified as "pink puffers" or "blue bloaters", ${ }^{78}$ or A, B and X types. ${ }^{9}$ The phenotypic classification of COPD we propose here differs from classic phenotypes as the prevalence of chronic bronchitis symptoms was almost equal in the three groups studied. Indeed, we used to see far more subjects with COPD who were suffering from chronic bronchitis in the past than currently. However, in Japan at least, we have seen a dramatic decrease in the number of subjects diagnosed with COPD and chronic bronchitis over the last three decades. ${ }^{32}$ Our data indicate that the decreasing frequency of chronic bronchitis in COPD over recent years does not reflect an increase of subjects with severe emphysema, but rather the manifestation of subjects with predominantly small airways disease which is not necessarily associated with bronchitis symptoms.

The reversibility of airflow limitation in COPD has long been a subject of debate. ${ }^{33-35}$ In this study we found a wide variation between subjects in BDR to salbutamol, but no statistical differences according to phenotype based on severity of emphysema. These data led to two important speculations. First, conclusions of the present study were unlikely to be biased by the inadvertent inclusion of patients with bronchial asthma, particularly in the group with no/mild emphysema. Second, what is occurring in large or proximal airways (chronic bronchitis symptoms and reversibility of airflow limitation) may be independent of what is occurring in peripheral sites in the lungs (small airways disease and emphysema).

There are two limitations to this study. First, we used subjective visual scoring for assessment of emphysema severity rather than objective quantification. This is because we had to use various kinds of CT scanners for this study and could not obtain the Digital Imaging and Communications in Medicine (DICOM) images from all affiliated hospitals. However, all HRCT images were thin-slice $(<2 \mathrm{~mm})$ and we carefully optimised the data acquisition parameters as well as the parameters for data analysis at each hospital to obtain ideal images for assessment of emphysema. In addition, we showed that a visual emphysema score for three CT images was highly correlated with objective volume-based computerised assessment for the whole lung in almost half the subjects, and also found a significant correlation between the visual emphysema score and TLCO/VA as described previously. ${ }^{36}$ Second, this study did not directly evaluate small airways disease, so we could not measure the real contribution of small airways disease to airflow limitation in any subjects. In parallel with this study, we attempted to develop new computer software using curved multiplanar reconstruction to obtain longitudinal images and to analyse accurately short-axis images of airways with inner diameter $\geqslant 2 \mathrm{~mm}$ located anywhere in the lungs. We recently published a paper which showed that airflow limitation in COPD is more closely related to the dimensions of the distal airways (sixth generation) than the proximal airways (third generation) in both upper and lower lobes. ${ }^{37}$ The observed high correlation coefficients between $\mathrm{FEV}_{1}$ percentage predicted and the dimensions of such distal airways are in sharp contrast to the weak but significant relationship between $\mathrm{FEV}_{1}$ percentage predicted and severity of emphysema observed in this study. These data suggest that the site of small airways contributes more significantly to airflow limitation than emphysema in COPD. In other words, the contribution of the small airways may be vitally important in COPD regardless of the phenotype based on severity of emphysema.

In conclusion, this study has shown that the severity of emphysema is highly variable, even among subjects with the same stage of COPD, and that COPD phenotypes based on severity of emphysema clearly differ from the classic phenotypes of "pink puffers" and "blue bloaters". The prevalence of bronchitis symptoms and average bronchodilator responses to inhaled $\beta_{2}$ agonist were similar among the three groups classified according to severity of emphysema. However, the BMI was significantly lower and SGRQ scores were significantly worse in the phenotype with severe emphysema than in those with no/mild emphysema. Accordingly, classification of COPD based on CT scanning may provide distinct phenotypes and display great clinical relevance in the management of COPD. Further studies are ongoing in an attempt to examine possible differences in the natural history of the disease according to phenotypes based on the severity of emphysema.

\section{ACKNOWLEDGEMENTS}

The authors thank Keimi Matsuura, Atsuko Mihara, Miki Ikeda, Hideka Ashikaga and Yuko Takagi at the Central Office of Hokkaido COPD Cohort Study, and the nurses and technicians in all affiliated hospitals involved in the study.

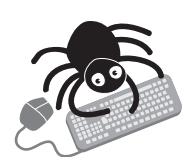

Further details are given in the online supplement available at http://thorax.bmj.com/supplemental.

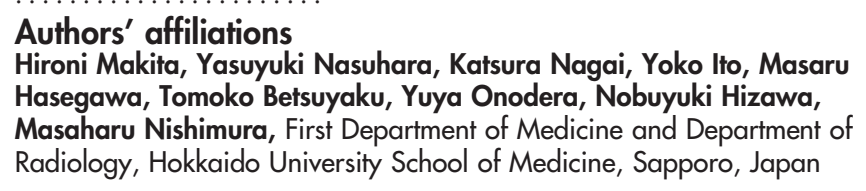

This study was supported by a scientific research grant from the Ministry of Education, Science, Culture and Sports of Japan (17390239 to MN), a grant to the Respiratory Failure Research Group from the Ministry of 
Health, Labor and Welfare, Japan and a research grant from Nippon Boehringer Ingelheim Co Ltd and Pfizer Japan Inc.

Competing interests: None.

Contributors: KKR Sapporo Medical Center: Yoshikazu Kawakami, Youichi Nishiura, Hiroshi Saito, Tetsuya Kojima, Kazuhiko Sakai, Yoriko Demura, Yukihiro Tsuchida, Motoko Tsubono, Kazuhiro Tsuboya, Shinichi Kakimoto; Iwamizawa Rosai Hospital: Takeshi Igarashi, Kiyonobu Kimura, Ikuo Nakano, Kouichi Itabashi, Kiyoshi Morikawa, Seiichi Tagami, Rika Sato, Junichiro Kojima, Shinii Nigawara, Shiro Fujii, Kazuyoshi Kanehira, Ryota Funakoshi, Yui Takashima, Masahiro Awaka, Hitoshi Ishii, Makoto Nakayama, Hiroki Honda, Ryo Kaneda, Masahisa Takagi; Sapporo City General Hospital: Hiroshi Yamamoto, Shigeaki Ogura, Kenii Akie, Kensuke Baba, Hiroki Goya, Kihoko Kitamura, Shiho Mineta, Takayo Takeda, Kiyoshi Kubo, Hiroshi Nara; Otaru City Hospital: Tsuyoshi Nakano, Kimihiro Takeyabu, Chihiro Naka, Hiroko Sato, Teiji Yamamoto, Toshio Abe; Hokkaido Social Insurance Hospital: Yasushi Akiyama, Fujiya Kishi, Akihide lto, Masashi Ooe, Michihiro Fujino, Yasuko Noda, Teruyo Takahashi, Keiko Abe, Mayumi Souma, Emiko Sato, Sumiyo Miyakawa, Tomokazu Indo, Shizuko Taguchi, Azusa Nakajima, Tomonori Fujii, Hironari Mori, Hideo Taguchi, Takashi Kojima, Ryouji Minami, Shigeki Murakami, Yuzuri Oono, Osamu Ishigamori, Satoru Akimoto, Takashi Emoto; Iwamizawa City General Hospital: Akira Kamimura, Toshiyuki Harada, Nobuyuki Hakuma, Eriko Anada, Tamaki Numata, Teiko Itakura, Tomoko lizawa, Yoshihiro Honoki; Sapporo Social Insurance General Hospital: Kazuo Takaoka, Isamu Doi, Miki Suzuki, Sachiko Komuro, Yoshiko Yoshida, Hitoshi Seki; Kinikyo Chuo Hospital: Atsushi Ishimine, Ryouji Nakano, Masako Ishihara, Fumiyo Itagaki, Naoya Matsuzaka, Takae Kosukegawa, Eriko Miyajima, Kimitsugu Nakamura, Wako Funayama, Katsumigi Tsuchiya, Ryouji Kaihatsu; Tenshi Hospital: Kaoru Kamishima, Yasushi Hasegawa; Hokkaido University Hospital/ Hokkaido University School of Medicine; First Department of Medicine: Motoko Kobayashi, Takeshi Hosokawa, Satoshi Fuke, Nao Odajima, Kunio Hamada, Eiji Shibuya, Yoshiko Obata, Kotomi Hosono, Kana Yoshikuni, Tomoko Akiyama; The Division of Pulmonary Function, the Department of Laboratory Medicine: Katsuaki Nitta, Masashi Yamamoto; Department of Health Science: Kenji Miyamoto.

\section{REFERENCES}

1 Global Initiative for Chronic Obstructive Lung Disease (GOLD). Global strategy for the diagnosis, management, and prevention of chronic obstructive pulmonary disease (updated 2003). Bethesda, MD: National Heart, Lung and Blood Institute, World Health Organization, 2003.

2 Fukuchi $Y$, Nishimura $M$, Ichinose $M$, et al. COPD in Japan: the Nippon COPD Epidemiology Study. Respirology 2004;9:458-65.

3 Lopez AD, Murray CC. The global burden of disease, 1990-2020. Nat Med 1998;4:1241-43.

4 Celli BR, MacNee W. ATS/ERS Task Force. Standards for the diagnosis and treatment of patients with COPD: a summary of the ATS/ERS position paper. Eur Respir J 2004;23:932-46.

5 American Thoracic Society/European Respiratory Society. Standards for the diagnosis and management of subjects with COPD, 2004. http:// www.ersnet.org/IrPresentations/copd/files/main/contenu/pages/full_text.pdf (accessed 2 September 2007).

6 Hogg JC. Pathophysiology of airflow limitation in chronic obstructive pulmonary disease. Lancet 2004;364:709-21.

7 Filley GF, Beckwitt HJ, Reeves JT, et al. Chronic obstructive bronchopulmonary disease. II. Oxygen transport in two clinical types. Am J Med 1968;44:26-38.

8 Netter F, Divertie M, Brass A. The CIBA collection of medical illustrations: Volume 7: Respiratory systems, CIBA Pharmaceutical Company, 1979

9 Burrows B, Fletcher CM, Heard BE, et al. The emphysematous and bronchial types of chronic airways obstruction. A clinicopathological study of patients in London and Chicago. Lancet 1966;1:830-5.
10 American Thoracic Society. Standards for the diagnosis and care of subjects with chronic obstructive pulmonary disease. Am J Respir Crit Care Med 1995; 152:S77-121.

11 Hogg JC, Macklem PT, Thurlbeck WM. Site and nature of airway obstruction in chronic obstructive lung disease. N Engl J Med 1968;278:1355-60.

12 Yanai M, Sekizawa K, Ohrui T, et al. Site of airway obstruction in pulmonary disease: direct measurement of intrabronchial pressure. J Appl Physiol 1992;72:1016-23.

13 Wright JL, Lawson LM, Pare PD, et al. The detection of small airways disease. Am Rev Respir Dis 1984;129:989-94.

14 Thurlbeck WM. The pathology of small airways in chronic airflow limitation. Eur J Respir Dis 1982;121(Suppl):9-18.

15 Hogg JC, Chu F, Utokaparch S, et al. The nature of small-airway obstruction in chronic obstructive pulmonary disease. N Engl J Med 2004;350:2645-53.

16 Ciba Guest Symposium Report. Terminology, definitions, and classification of chronic pulmonary emphysema and related condition. Thorax 1964;14:286-99.

17 Braman SS. Chronic cough due to chronic bronchitis: ACCP evidence-based clinical practice guidelines. Chest 2006;129:104-15S.

18 Committee of Pulmonary Physiology, the Japanese Respiratory Society Guidelines for pulmonary function tests: spirometry, flow-volume curve, diffusion capacity of the lung (in Japanese). Tokyo: Japanese Respiratory Society, 2004.

19 Goddard PR, Nicholson EM, Laszlo G, et al. Computed tomography in pulmonary emphysema. Clin Radiol 1982;33:379-87.

20 Jones PW, Quirk FH, Baveystock CM, et al. A self-complete measure of health status for chronic airflow limitation. The St George's Respiratory Questionnaire. Am Rev Respir Dis 1992;145:1321-7.

21 Gelb AF, Hogg JC, Muller NL, et al. Contribution of emphysema and small airways in COPD. Chest 1996;109:353-9.

22 Hogg JC, Wright JL, Wiggs BR, et al. Lung structure and function in cigarette smokers. Thorax 1994;49:473-8.

23 Gelb AF, Schein M, Kuei J, et al. Limited contribution of emphysema in advanced chronic obstructive pulmonary disease. Am Rev Respir Dis 1993;147:1157-61.

24 Celli BR, Cote CG, Marin JM, et al. The body-mass index, airflow obstruction, dyspnea, and exercise capacity index in chronic obstructive pulmonary disease N Engl J Med 2004;350:1005-12.

25 Anthonisen NR. Prognosis in chronic obstructive pulmonary disease: results from multicenter clinical trials. Am Rev Respir Dis 1989;140:S95-9.

26 Nishimura K, Izumi T, Tsukino M, et al. Dyspnea is a better predictor of 5-year survival than airway obstruction in patients with COPD. Chest 2002;121:1434-40.

27 Domingo-Salvany A, Lamarca R, Ferrer M, et al. Hearth-related quality of life and mortality in male patients with chronic obstructive pulmonary disease. Am J Respir Crit Care Med 2002;166:680-5.

28 Marti S, Munoz X, Rios J, et al. Body weight and comorbidity predict mortality in COPD patients treated with oxygen therapy. Eur Respir J 2006;27:689-96.

29 Yoshioka A, Betsuyaku T, Nishimura M, et al. Excessive neutrophil elastase in bronchoalveolar lavage fluid in subclinical emphysema. Am J Respir Crit Care Med 1995; 152:2127-32.

30 Betsuyaku T, Nishimura M, Takeyabu K, et al. Neutrophil granule proteins in bronchoalveolar lavage fluid from subjects with subclinical emphysema. Am J Respir Crit Care Med 1999;159:1985-91.

31 Takabatake N, Sata M, Inove S, et al. A novel polymorphism in secretory phospholipase A2-IID is associated with body weight loss in chronic obstructive pulmonary disease. Am J Respir Crit Care Med 2005;172:1097-104.

32 Japanese Respiratory Society. COPD guideline for diagnosis and treatment (in Japanese). Tokyo: Japanese Respiratory Society, 2004.

33 Anthonisen NR, Wright EC, the IPPB Trial Group. Bronchodilator response in chronic obstructive pulmonary disease. Am Rev Respir Dis 1986;133:814-9.

34 Calverley PM, Burge PS, Spencer S, et al. Bronchodilator reversibility testing in chronic obstructive pulmonary disease. Thorax 2003;58:659-64.

35 Anthonisen NR, Lindgren PG, Tashkin DP, et al. Lung Health Study Research Group. Bronchodilator response in the lung health study over 11 yrs. Eur Respir J 2005;26:45-51.

36 Gould GA, Redpath AT, Ryan M, et al. Lung CT density correlates with measurements of airflow limitation and the diffusing capacity. Eur Respir $J$ $1991 ; 4: 141-6$.

37 Hasegawa M, Nasuhara $Y$, Onodera $Y$, et al. Airflow limitation and airway dimensions in chronic obstructive pulmonary disease. Am J Respir Crit Care Med 2006;173:1309-15. 